\title{
Characterizing acyl-carnitine biosignatures for schizophrenia: a longitudinal pre- and post-treatment study
}

\author{
Bing Cao', Dongfang Wang ${ }^{1}$, Zihang Pan², Elisa Brietzke ${ }^{2,3}$, Roger S. Mclntyre ${ }^{2,4}$, Natalie Musial $\mathbb{C}^{2}$, Rodrigo B. Mansur²,
} Mehala Subramanieapillai ${ }^{2}$, Jing Zeng ${ }^{1,5,6}$, Ninghua Huang ${ }^{1,5,6}$ and Jingyu Wang ${ }^{1,5,6}$

\begin{abstract}
Subjects with schizophrenia have high risks of metabolic abnormalities and bioenergetic dysfunction. Acyl-carnitines involved in bioenergetic pathways provide potential biomarker targets for identifying early changes and onset characteristics in subjects with schizophrenia. We measured 29 acyl-carnitine levels within well-characterized plasma samples of adults with schizophrenia and healthy controls using liquid chromatography-mass spectrometry (LC-MS). Subjects with schizophrenia were measured at baseline and after 8 weeks of treatment. A total of 225 subjects with schizophrenia and 175 age- and gender-matched healthy controls were enrolled and 156 subjects completed the 8week follow-up. With respect to plasma acyl-carnitines, the individuals with schizophrenia at baseline showed significantly higher levels of $\mathrm{C} 4-\mathrm{OH}$ (C3-DC) and C16:1, but lower concentrations of C3, C8, C10, C10:1, C10:2, C12, $\mathrm{C} 14: 1-\mathrm{OH}, \mathrm{C14}: 2$, and $\mathrm{C} 14: 2-\mathrm{OH}$ when compared with healthy controls after controlling for age, sex, body mass index (BMI), smoking, and drinking. For the comparison between pretreatment and posttreatment subjects, all detected acylcarnitines were significantly different between the two groups. Only the concentration of C3 and C4 were increased after selection by variable importance in projection (VIP) value $>1.0$ and false discovery rate (FDR) $q$ value $<0.05$. A panel of acyl-carnitines were selected for the ability to differentiate subjects of schizophrenia at baseline from controls, pre- from post-treatment, and posttreatment from controls. Our data implicated acyl-carnitines with abnormalities in cellular bioenergetics of schizophrenia. Therefore, acyl-carnitines can be potential targets for future investigations into their roles in the pathoetiology of schizophrenia.
\end{abstract}

\section{Introduction}

Schizophrenia is a severe and complex lifelong mental disorder associated with significant morbidity and mortality ${ }^{1}$. The prevalence of schizophrenia is between 0.5 and $1.0 \%$ worldwide ${ }^{1,2}$. Schizophrenia is a multifaceted mental illness with both genetic and environmental etiology, characterized by psychopathology, as well as cognitive and neurobiological abnormalities ${ }^{3}$. Despite

Correspondence: Jingyu Wang (wjy@bjmu.edu.cn)

${ }^{1}$ Department of Laboratorial Science and Technology, School of Public Health, Peking University, Beijing 100191, P. R. China

${ }^{2}$ Mood Disorders Psychopharmacology Unit, Toronto Western Hospital, University Health Network, Toronto, ON, Canada

Full list of author information is available at the end of the article. decades of research, the underling pathogenic pathways underlying schizophrenia has yet to be comprehensively understood. It has been previously established that subjects with schizophrenia have relatively higher rates of metabolic abnormalities and bioenergetic dysfunction (i.e., metabolic syndrome, obesity, and type 2 diabetes mellitus ${ }^{4-6}$. Further studies suggest that schizophrenia can be characterized by molecular targets, thus presenting opportunities to identify metabolic abnormalities before the initiation of treatment ${ }^{7,8}$.

Carnitines are essential dietary nutrients obtained from meat and dairy products, or are endogenously biosynthesized from L-lysine and L-methionine in the liver and kidneys ${ }^{9,10}$. The optically-active isomer L-carnitine is

\section{(c) The Author(s) 2019}

(c) Open Access This article is licensed under a Creative Commons Attribution 4.0 International License, which permits use, sharing, adaptation, distribution and reproduction cc) in any medium or format, as long as you give appropriate credit to the original author(s) and the source, provide a link to the Creative Commons license, and indicate if changes were made. The images or other third party material in this article are included in the article's Creative Commons license, unless indicated otherwise in a credit line to the material. If material is not included in the article's Creative Commons license and your intended use is not permitted by statutory regulation or exceeds the permitted use, you will need to obtain permission directly from the copyright holder. To view a copy of this license, visit http://creativecommons.org/licenses/by/4.0/. 
present in mammalian cells as both free carnitine and acyl-carnitines ${ }^{11}$. Accumulating evidence from animal and human studies indicate that carnitines are quaternary ammonium molecules involved in many metabolic pathways. A recent review paper summarized that acylcarnitines play an essential role in transporting longchain fatty acids across the mitochondrial inner membrane during $\beta$-oxidation, which increases antioxidant activity, stimulates the activity of certain proteins, and enhances cholinergic neurotransmission ${ }^{11}$. Fatty acid metabolism is intricately involved with energy bioenergetics and oxidative pathways ${ }^{11,12}$. Given that carnitines are implicated in fatty acid oxidation, energy production, and cellular homeostasis pathways, the dysfunction of carnitines would be expected to produce a wide variety of disorders affecting the functions of several organs, including, but not limited to, liver, skeletal muscle, heart, and brain ${ }^{13,14}$. A recent animal study with healthy mice reported that chronic acetyl-L-carnitine intake alters brain energy metabolism and increases norepinephrine and serotonin content ${ }^{15}$. In human health and disease research, acyl-carnitine profiles have been widely used to identify metabolic perturbations ${ }^{11}$, including inborn errors of metabolism ${ }^{16}$, thyroid dysfunction ${ }^{17}$, and type 2 diabetes $^{18}$. Carnitine and acyl-carnitine supplementation have also been used in the treatment of various illnesses (e.g., Alzheimer's disease, peripheral arterial disease, and celiac disease) for decades with the primary function of regulating cellular bioenergetics ${ }^{19,20}$. Furthermore, accumulated evidence indicates that alterations of carnitines may contribute to certain mental illnesses, including depression $^{21}$, autism spectrum disorder ${ }^{22}$, and schizophrenia $^{23}$. In particular, several recent studies have reported that schizophrenia is associated with an imbalance in redox or oxidative stress status. Current replicated evidence suggests that acyl-carnitines are involved in bioenergetics, $\beta$-oxidation, and tricarboxylic acid cycle regulation in the mitochondrion. These mechanism could play important roles in the pathogenesis of schizophrenia ${ }^{24,25}$. In addition, acetyl-carnitine and levocarnitine ${ }^{26,27}$ supplementation therapies have been used to target carnitine deficiency observed in individuals with schizophrenia. Available evidence suggest that acylcarnitines impact insulin sensitivity, lipid metabolism, inflammatory response, as well as bioenergetic functions $^{23,28}$. Based on these foregoing evidence, we believe acyl-carnitines play an essential role in the pathoetiology of schizophrenia. Thus, a comprehensive analysis of acylcarnitine profiles in a treatment population of individuals with schizophrenia was conducted.

The hypothesis of the current study is that bioenergetic abnormalities defined by acyl-carnitine imbalance, exist in subjects with schizophrenia. By utilizing a targeted metabolic profiling approach, our current study was able to accurately quantify acyl-carnitine profiles in plasma samples among individuals with schizophrenia at baseline and after 8 weeks of antipsychotic therapy. The same metabolic profiling approach was applied to the healthy control subjects. Herein, our study aimed to find potential abnormalities of metabolic pathways involving acylcarnitines to further explore the connection between schizophrenia and metabolism.

\section{Methods \\ Study population}

Two hundred twenty-five individuals with a primary diagnosis of schizophrenia according to the Diagnostic and Statistical Manual of Mental Disorders, fourth edition (DSM-IV) were enrolled. All subjects were inpatients from the Mental Health Center of Weifang (Shandong Province, China). All subjects received antipsychotic treatment and were invited to an 8-week follow-up survey. Subjects received antipsychotic therapies according to their clinical performance. Among the 225 subjects enrolled, 69 withdrew or were lost to follow-up. During the same time period, 175 healthy individuals from the same city district as the subjects with schizophrenia, that are age ( \pm 5 years), sex, and ethnicity matched, without a history of DSM-defined psychiatric disorders, were enrolled as a control comparison group. All subjects met the following criteria: (1) age from 18 to 60 years old; (2) absence of diabetes mellitus, hyperlipidemia, cardiovascular disease, or any other severe or unstable medical illness; (3) absence of comorbidity with other psychiatric disorders, including alcohol and substance use disorders. All subjects were either first psychotic episode and drugnaive or had recurrent schizophrenia and had not taken any antipsychotic drugs for a minimum of 4 weeks before hospitalization. Clinical assessments took place between November 2015 and September 2016.

Data on basic demographic characteristics was collected by a standardized questionnaire. The Case Report Form (CRF) was used to collect clinical data, clinical history, family history, and medication records. The Positive and Negative Syndrome Scale (PANSS) scores were assessed during admission/before treatment (week 0 ) and after treatment (week 8). Treatment medication details are shown in Table S1. During the trial, all subjects received a standard diet provided by the hospital and abstained from alcohol and smoking. For the control group, basic clinical information and blood samples were collected for one time only (i.e., at week 0 ).

\section{Ethical approval}

The research ethics committee of the Peking University Health Science Center (Beijing, China) reviewed and approved the study protocols (IRB00001052-14071). All subjects provided written informed consent prior to their 
inclusion. All procedures were performed in accordance with the Helsinki Declaration standards as revised in 1989.

\section{Plasma samples preparation}

All fasting blood samples $(\sim 5 \mathrm{~mL})$ were collected from subjects at baseline (week 0) before initiation of antipsychotic treatment and after 8 weeks of antipsychotic treatment following overnight fasting $(12 \mathrm{~h}$ fasting, collected between 7 and $9 \mathrm{am}$ ). Samples were maintained at $4{ }^{\circ} \mathrm{C}$ for $20-30 \mathrm{~min}$ and the resulting plasma aliquot was transferred into Eppendorf tubes, which were subsequently stored at $-80^{\circ} \mathrm{C}$. The plasma samples of the control group were collected with the same protocols as the schizophrenia group.

\section{Chemicals and reagents}

Analytical reference standards of 29 acyl-carnitines (Sigma-Aldrich, Co., St. Louis, MO, USA) were employed for quality control of compounds in samples. We defined acyl-carnitines and major acyl-carnitine fractions as free-, short-, medium-, or long-chain acylcarnitines according to the length of carbon chains in the molecular structure (i.e., free carnitine: $\mathrm{C} 0$, short-chain: C2-C5, medium-chain: C6-C12, long-chain: C14$\mathrm{C} 18)^{29,30}$. Deuterium-labeled carnitine and acyl-carnitines (NSK-B Set, Cambridge Isotope Laboratories, Inc., Tewksbury, MA, USA) were used as internal standards, including ${ }^{2} \mathrm{H}_{9}$-carnitine (free carnitine, $\mathrm{CN}$ ), ${ }^{2} \mathrm{H}_{3}$-acetylcarnitine (C2), ${ }^{2} \mathrm{H}_{3}$-propionylcarnitine (C3), ${ }^{2} \mathrm{H}_{3}$-butyrylcarnitine (C4), ${ }^{2} \mathrm{H}_{9}$-isovalerylcarnitine (C5), ${ }^{2} \mathrm{H}_{3}-$ octanoylcarnitine (C8), ${ }^{2} \mathrm{H}_{9}$-myristoylcarnitine (C14), and ${ }^{2} \mathrm{H}_{3}$-palmitoylcarnitine (C16). HPLC-grade ammonium acetate was purchased from Sigma-Aldrich (St. Louis, MO, USA); HPLC-grade formic acid and methanol were supplied by Thermo Fisher Scientific (Waltham, MA, USA); pure water was purchased from Wahaha Co., Ltd. (Hangzhou, China).

\section{Carnitine and acyl-carnitines extraction from plasma samples}

The deuterium-labeled carnitine and acyl-carnitines were initially redissolved in $1 \mathrm{~mL}$ methanol as stock solution for internal standards quantification. Mixtures of $45 \mu \mathrm{L}$ thawed plasma and $135 \mu \mathrm{L}$ of methanol solution $(1: 3, \mathrm{v} / \mathrm{v})$ spiked with $5.4 \mu \mathrm{L}$ of isotopically labeled internal standards $(\mathrm{v} / \mathrm{v}=24 / 1)$ were vibrated and centrifuged $\left(4{ }^{\circ} \mathrm{C}, 12,000 \mathrm{rpm}\right.$ for $\left.10 \mathrm{~min}\right)$ and $100 \mu \mathrm{L}$ of supernatant was collected into a $200 \mu \mathrm{L}$ vial insert for analysis. Equal volumes of the supernatant from all samples were transferred each time to a limited volume vial. Pooled quality control samples (QCs) consisted of small aliquots of each biological sample being pooled and mixed together to monitor the stability and repeatability of the results.

\section{HILIC mode and UHPLC-MS analysis}

Plasma acyl-carnitine profiles were measured in a randomized order. The UPLC separation was performed using the Thermo Scientific ${ }^{\mathrm{TM}}$ Dionex $^{\mathrm{TM}}$ UltiMate $^{\mathrm{TM}} 3000$ Rapid Separation LC (RSLC) system with a BEH Amide column $(2.1 \times 100 \mathrm{~mm}, 1.7 \mu \mathrm{m}$; Waters Corp $)$. Samples were analyzed using a positive ionization mode (ESI+) with the following settings: the column flow rate was $300 \mu \mathrm{L} / \mathrm{min}$, the column temperature was set at $50{ }^{\circ} \mathrm{C}$, the auto-sampler temperature was kept at $4{ }^{\circ} \mathrm{C}$, and the injection volume was $1 \mu \mathrm{L}$. The working solvent $\mathrm{A}$ was methanol and solvent $B$ was ultrapure water; both $A$ and $B$ contained $0.1 \%$ formic acid and $10 \mathrm{mmol} / \mathrm{L}$ ammonium acetate. The separation gradient used was: $0-1 \mathrm{~min}(95.0 \%$ A), 1-10 min: (95.0-50.0\% A), 10-12 min: (50.0\% A), 12-12.1 $\mathrm{min}$ (50.0-95.0\% A), and 12.1-16 min: (95.0\% A).

After the chromatographic separation, MS ionization and data acquisition were performed using a Thermo Scientific $^{\mathrm{TM}}$ Q Exactive ${ }^{\mathrm{TM}}$ hybrid quadrupole Orbitrap mass spectrometer equipped with a HESI-II probe. The HESI-II spray voltages were $3.7 \mathrm{kV}$. The heated capillary temperature was $320^{\circ} \mathrm{C}$, the sheath gas pressure was $30 \mathrm{psi}$, the auxiliary gas setting was $10 \mathrm{psi}$, and the heated vaporizer temperature was $300^{\circ} \mathrm{C}$. Both the sheath gas and the auxiliary gas were nitrogen. The collision gas was also nitrogen at a pressure of $1.5 \mathrm{~m}$ Torr. The parameters for the full mass scan were as follows: a resolution of 70,000 , an auto gain control target under $1 \times 10^{6}$, a maximum isolation time of $50 \mathrm{~ms}$, and an $\mathrm{m} / \mathrm{z}$ range of 70-1050. The calibration was customized for the Q Exactive to keep the mass tolerance of $5 \mathrm{ppm}$. The parameters for the QC-MS2 scan were as follows: a resolution of 17,500 , an auto gain control target under $1 \times 10^{5}$, a maximum isolation time of $50 \mathrm{~ms}$, a loop count of top 10 peaks, an isolation window of $m / z 2$, a normalized collision energy of $30 \mathrm{~V}$, and an intensity threshold under $1 \times$ $10^{5}$. The final LC-MS method employed for the quantification of carnitines and internal standards was composed of 29 carnitines and 8 internal standards ESI+ timed functions properly segmented over the $10 \mathrm{~min}$ chromatographic run. The HILIC UPLC-MS system control and data acquisition were performed with the Thermo Fisher Corporation Xcalibur 2.2 SP1.48 software (Waltham, MA). Skyline (64-bit, 3.5.0.9319, MacCoss Lab, UW) was used to visualize, process, and interpret the MS raw data, allowing for the discovery and identification of carnitines among sample groups.

\section{Statistical analysis}

All plasma acyl-carnitine levels were quality reassured by analytical reference standards, quantified and normalized by its corresponding internal standard level (Table S2), and $\log _{10}$-transformed to approximate normality. Descriptive statistics were performed for the basic 
characteristics and acyl-carnitine analysis consisted of categorical variables summarized as frequencies and proportions, while continuous variables were summarized as the mean and standard deviation (SD) or median and interquartile range (IQR). For categorical variables, statistical significance between various groups was tested using the $\chi^{2}$ test. For continuous variables, according to the features of data, the independent Student's $t$-test or Mann-Whitney $\mathrm{U}$ test was used for comparisons between the two independent groups (i.e., control and patient groups, or first and recurrent subjects). The paired Student's $t$-test or Wilcoxon signed-rank $t$-test was employed for comparisons between the two related groups (i.e., preand post-treatment groups). A two-sided $P<0.05$ was considered as statistically significant. The Benjamini-Hochberg false discovery rate (FDR) control was implemented to correct for multiple comparisons of 29 acyl-carnitines. In this study, the FDR $q$-value threshold for significant markers was set at 0.05 .

Orthogonal partial least-squares discriminant analysis (OPLS-DA) was performed using $\log _{10}$-transformed and auto-scaled data to construct classification models. An internal 7-fold cross-validation was carried out to estimate the performance of the OPLS-DA model. Model validation was performed using 300 -iteration permutation tests. $R^{2}$ represents the explanation capacity of the model, while $Q^{2}$ stands for the predictive capacity of the model. OPLS-DA model was performed by the SIMCA-P software 14.1 (Umetrics, Umeå, Sweden).

Based on the $q$ value $<0.05$ from univariate analysis and the highest variable importance in projection (VIP) value $>1.0$ from the OPLS-DA model, several metabolites were selected as a panel of markers to differentiate subjects of pretreatment, posttreatment patients, and healthy controls. Partial correlation analysis on ranks (Spearman Correlation) was used to calculate correlation coefficients among acyl-carnitines and between clinical characteristics with acyl-carnitines, which was conducted by Metaboanalyst 3.0 software. Generalized linear models (GLM) were applied to comparisons of carnitines' levels between two groups with adjustment for confounding factors. According to the previous metabolic profiling research $^{31,32}$, age, sex, BMI, smoking, and drinking were defined as potential confounders in our data analysis. Due to the potential linearity and collinearity, integrating individual carnitine was done one at a time into the GLM. Area under the receiver-operating characteristic (ROC) curve (AUROC) was then calculated to evaluate its classification performance using SPSS 22.0 (SPSS Inc., Chicago, IL, USA). Backward stepwise logistic regression models based on Akaike's information criterion (AIC) ${ }^{33}$ were also performed by SPSS 22.0 to optimize the metabolite biomarker combination for discrimination between pretreatment and posttreatment schizophrenia subjects, as well as healthy controls.

\section{Results}

Demographic and clinical characteristics of the sample

In total, 225 individuals with schizophrenia and 175 healthy controls were included in the analysis. Demographic data and clinical characteristics were compared between individuals with schizophrenia at baseline and healthy controls (Table 1). Individuals at baseline with schizophrenia were younger than controls $(P=0.04)$. The mean age of subjects and controls were 37.31 years $(\mathrm{SD}=$ 10.85 years) and 39.44 years $(\mathrm{SD}=9.36$ years), respectively. In terms of gender distribution, $60.0 \%$ of the subjects and $69.1 \%$ of the healthy controls were female. Of the individuals with schizophrenia, the mean age of onset was 22.74 $(\mathrm{SD}=9.70)$ years, while the mean duration of illness was $4.48(\mathrm{SD}=2.67)$ years. Of the 225 subjects with schizophrenia, 40 were first-episode and treatmentnaive, while the remaining 185 schizophrenia subjects were recurrent (had psychotic episodes in the past) and had not taken any antipsychotic drugs for at least 1 month prior to hospitalization. No significant changes in BMI, fasting blood glucose (FBG), total cholesterol (TC), triglycerides (TG) and very low-density lipoprotein (VLDL) were observed between the two groups (all $P>0.05$ ). The comparison of all basic characteristics between first

Table 1 Baseline characteristics of subjects with schizophrenia at baseline and healthy control

\begin{tabular}{lccc}
\hline Variables & $\begin{array}{l}\text { Schizophrenia } \\
\boldsymbol{n}=\mathbf{2 2 5}\end{array}$ & $\begin{array}{c}\text { Control } \\
\boldsymbol{n}=\mathbf{1 7 5}\end{array}$ & $\boldsymbol{P}$ value \\
\hline Age (years); mean (SD) & $37.31(10.85)$ & $39.44(9.36)$ & $0.040^{\mathrm{a}}$ \\
Gender (female); $n$ (\%) & $135(60.0)$ & $121(69.1)$ & $0.061^{\mathrm{b}}$ \\
Smoker; $n$ (\%) & $25(11.3)$ & $21(12.0)$ & $0.936^{\mathrm{b}}$ \\
Drinker; $n$ (\%) & $10(5.0)$ & $30(17.4)$ & $<0.001^{\mathrm{b}}$ \\
Age of onset (years); mean (SD) & $27.74(9.70)$ & - & - \\
Duration of illness (years); mean & $4.48(2.67)$ & - & - \\
(SD) & & & \\
First-episode subjects; $n$ (\%) & $40(17.78)$ & - & - \\
BMI (kg/m²); mean (SD) & $24.11(3.85)$ & $23.91(3.14)$ & $0.596^{\mathrm{a}}$ \\
FBG (mmol/L); mean (SD) & $5.73(1.78)$ & $5.70(1.02)$ & $0.869^{\mathrm{a}}$ \\
TG (mmol/L); mean (SD) & $1.25(0.9)$ & $1.17(0.8)$ & $0.354^{\mathrm{a}}$ \\
TC (mmol/L); mean (SD) & $4.66(1.12)$ & $4.76(0.97)$ & $0.368^{\mathrm{a}}$ \\
VLDL (mmol/L); mean (SD) & $0.58(0.41)$ & $0.54(0.35)$ & $0.308^{\mathrm{a}}$ \\
\hline
\end{tabular}

$B M I$ body mass index, FBG fasting blood glucose, TG triglyceride, $T C$ total cholesterol, VLDL very low density lipoprotein, $S D$ standard deviation

a $P$ values were calculated by two-tailed $t$-tests

${ }^{b} P$ values were calculated by chi-square tests 
Table 2 Demographic and clinical characteristics of pretreatment and posttreatment subjects

\begin{tabular}{llll}
\hline Variables & $\begin{array}{l}\text { Pretreatment } \\
\boldsymbol{n}=\mathbf{1 5 6}\end{array}$ & $\begin{array}{l}\text { Posttreatment } \\
\boldsymbol{n}=\mathbf{1 5 6}\end{array}$ & $\boldsymbol{P}^{\text {value }} \mathbf{a}^{\mathbf{2}}$ \\
\hline PANSS scores; mean (SD) & & & \\
PANSS total & $88.82(18.23)$ & $50.67(12.7)$ & $<\mathbf{0 . 0 0 1}$ \\
PANSS positive & $21.78(8.09)$ & $9.96(4.00)$ & $<\mathbf{0 . 0 0 1}$ \\
PANSS negative & $21.15(7.99)$ & $13.65(5.25)$ & $<\mathbf{0 . 0 0 1}$ \\
General psychopathology & $42.84(12.49)$ & $25.67(7.94)$ & $<\mathbf{0 . 0 0 1}$ \\
BMI (kg/m²); mean (SD) & $23.89(3.96)$ & $24.70(3.70)$ & $<\mathbf{0 . 0 0 1}$ \\
Waist (cm); mean (SD) & $88.71(12.19)$ & $90.63(11.20)$ & $<\mathbf{0 . 0 0 1}$ \\
FBG (mmol/L); mean (SD) & $5.62(1.80)$ & $5.21(1.00)$ & $\mathbf{0 . 0 0 5}$ \\
TG (mmol/L); mean (SD) & $1.24(0.82)$ & $1.96(1.15)$ & $<\mathbf{0 . 0 0 1}$ \\
TC(mmol/L); mean (SD) & $4.61(1.08)$ & $4.63(0.92)$ & 0.821 \\
HDL (mmol/L); mean (SD) & $1.42(0.30)$ & $1.35(0.32)$ & $\mathbf{0 . 0 1 2}$ \\
LDL (mmol/L); mean (SD) & $2.25(0.45)$ & $2.29(0.46)$ & 0.295 \\
VLDL (mmol/L); mean (SD) & $0.57(0.38)$ & $0.91(0.52)$ & $<\mathbf{0 . 0 0 1}$ \\
\hline
\end{tabular}

$B M I$ body mass index, FBG fasting blood glucose, TG triglyceride, TC total cholesterol, $H D L$ high-density lipoprotein, $L D L$ low density lipoprotein, VLDL very low density lipoprotein, $S D$ standard deviation

${ }^{\text {a }} P$ values were calculated by two-tailed paired-samples

episode and recurrent episode patients before and after 8week follow-up are shown in Table S4.

Before the 8-week follow-up point, 69 of the 225 subjects withdrew from the study (either refused to follow-up or lost contact) at various points. Comparison of the clinical characteristics of the remaining 156 pretreatment and posttreatment subjects was completed after 8 weeks (Table 2). The scores of all PANSS domains were significantly decreased after treatment $(P<0.05)$ indicating a reduction of schizophrenia severity. No significant change from baseline in TC and low-density lipoprotein (LDL) were observed after the follow-up at 8 weeks (all $P>0.05$ ), while the BMI, waist circumference, TG, and VLDL were increased after treatment. FBG and high-density lipoprotein (HDL) were decreased after treatment.

\section{Plasma acyl-carnitine profiles of subjects}

Plasma acyl-carnitine levels of each group are illustrated in Fig. 1 and Table S3. All OPLS-DA models between the two groups indicated adequate classification (Fig. 2a-e). There were 11 out of 29 acyl-carnitines altered in subjects with schizophrenia at baseline compared to controls (FDR $q<0.05$ and OPLS-DA VIP $>1.0$, shown in Table 3). The subjects with schizophrenia at baseline showed significantly higher levels of C4-OH(C3-DC) and C16:1, but lower concentrations of $\mathrm{C} 3, \mathrm{C} 8, \mathrm{C} 10, \mathrm{C} 10: 1, \mathrm{C} 10: 2, \mathrm{C} 12$, C14:1-OH, C14:2, and C14:2-OH. All the differences still have statistical significance after controlling for age, sex,
BMI, smoking, and drinking. After administration of the 8-week clinical therapy, all detected acyl-carnitines had significant differences between the pre- and posttreatment groups with FDR $q<0.05$. Thirteen acylcarnitines have VIP $>1.0$ with the OPLS-DA selection. Only the concentration of $\mathrm{C} 3$ and $\mathrm{C} 4$ were increased while all the remaining 11 acyl-carnitines were decreased. We also conducted the comparison between posttreatment patients and controls to explore the difference. Our results indicated that all the fourteen selected acylcarnitines with FDR $q<0.05$ and OPLS-DA VIP $>1.0$ (i.e., C2, C8, C10, C10:1, C10:2, C12, C12:1, C14:1, C14:1$\mathrm{OH}, \mathrm{C} 14: 2, \mathrm{C} 14: 2-\mathrm{OH}, \mathrm{C} 16: 2, \mathrm{C} 16: 2-\mathrm{OH}$, and $\mathrm{C} 18)$ were lower in posttreatment patients after controlling age, sex, BMI, smoking, and drinking (Tables 3 and 4). To validate the reliability of the prediction model, permutation test $(n$ $=300$ ) was calculated (Fig. 2b-f). The true-class $\mathrm{Q}^{2}$ and $R^{2}$ values were significantly higher than the corresponding permutated values in all three figures, indicating that the model is statistically sound; that its high predictability is not due to over-fitting of the data.

Multivariate analysis for recurrent and first episode subjects showed only C3, C4, and C16 were higher in the recurrent schizophrenia group at baseline; $\mathrm{C} 3, \mathrm{C} 4$, and $\mathrm{C} 5$ were higher in the recurrent group after 8-week followup, with $P$ values $<0.05$ after adjusting for age, sex, BMI, smoking, and drinking. After FDR correction, all the acylcarnitines expressed $q$-values $>0.05$ at baseline and 8week follow-up between the first-episode and recurrent subject groups. These results demonstrate that recurrent subjects after at least 1 month without any antipsychotic drugs show similar plasma acyl-carnitine profiles to firstepisode subjects both at baseline and after 8-week followup (Table S4).

\section{Acyl-carnitine panel with best classification performance}

The ROC curves were carried out to explore the diagnostic effectiveness of all significant acyl-carnitines between specific groups. In this study, two groups of acyl-carnitines were screened by backward stepwise logistic regression models, which were based on Akaike's information criterion (AIC). For differentiating subjects with schizophrenia from controls at baseline, a combination of seven acyl-carnitines, including $\mathrm{C} 4 \mathrm{OH}$ (C3DC), C8, C10:1, C12, C14:1OH, C14:2, and C16:1, provided the best classification performance with the $\mathrm{AUC}=0.926$ (95\% CI: 0.901-0.952). For differentiating pretreatment from posttreatment subjects, a set of ten acyl-carnitines, including C2, C4, C4OH (C3DC), C10:1, C14:1, C14:1OH, C14:2, C16:1OH, C16:2, and C18:1, was used to build the ROC curve with the AUC $=0.851$ (95\% CI: 0.810-0.892), which indicated a high accuracy in predicting different groups. Nine acyl-carnitines provided the best classification performance between posttreatment patients and 

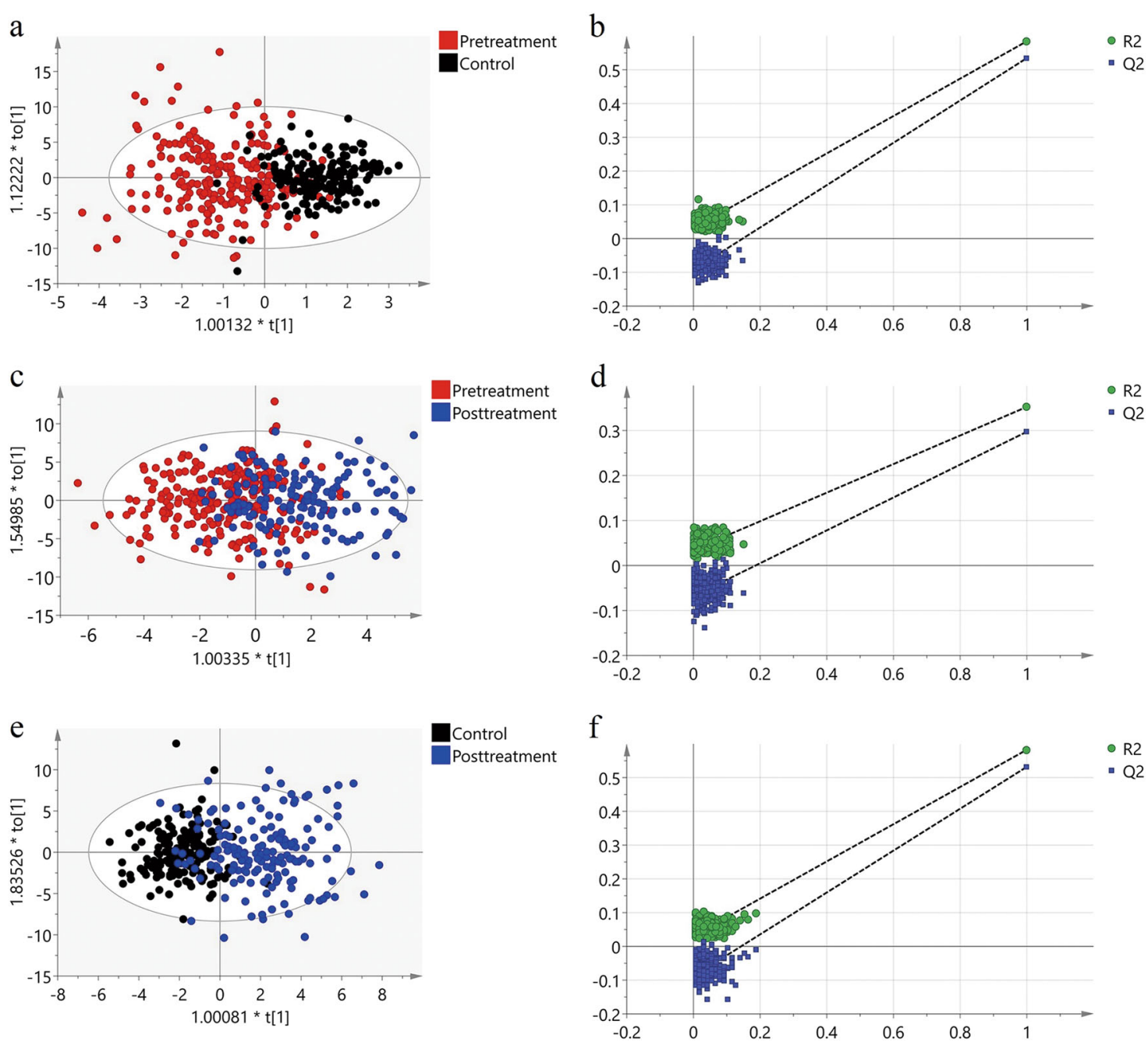

Fig. 1 Box plots of differential carnitines for the comparison between subjects at baseline and controls or upon treatment. The $X$-axis is the acyl-carnitines, and the $Y$-axis is the concentration of acyl-carnitines $(\mu \mathrm{mol} / \mathrm{L})$. * Statistically significant with $P<0.05$ in the comparison between subjects at baseline and controls. "Statistically significant with $P<0.05$ in the comparison between posttreatment subjects and controls

controls (i.e., C2, C8, C10:1, C12, C12:1, C14:1OH, $\mathrm{C} 14: 2 \mathrm{OH}, \mathrm{C} 16: 2 \mathrm{OH}$, and $\mathrm{C} 18)$ with $\mathrm{AUC}=0.950(95 \%$ CI: $0.928-0.972$ ) (Fig. S1).

\section{Correlations of acyl-carnitines and clinical outcomes}

The correlation analysis of acyl-carnitine concentrations was calculated using Spearman correlation analysis, considering subjects both before and after clinical treatment and healthy controls. As shown in Fig. 3, most acylcarnitines were correlated with each other, especially medium- and long-chain acyl-carnitines. The correlation coefficients among medium- and long-chain acyl-carnitines were strong, with almost all demonstrating $\mathrm{R}>0.6$ (data available under request). We also performed the correlation analysis of each group. The correlation coefficients of acyl-carnitines were similar in subjects at baseline, after follow-up, and in healthy controls (Figure $3)$.

Spearman correlation analysis was further applied to examine associations between the baseline levels of the acyl-carnitines and follow-up changes in the clinical variables (Fig. S3). BMI and waist circumference changes were negatively related to several short-chain and medium-chain acyl-carnitines at baseline $(P<0.05)$. Levels of C3 and C5 at baseline were inversely associated with 8-week follow-up changes in FBG among subjects. However, no significant difference was found between acyl-carnitine levels at baseline with regards to the changes of PANSS total scores.

\section{Discussion}

By using metabolomic techniques, our study is the first to accurately quantify acyl-carnitines in relation to 


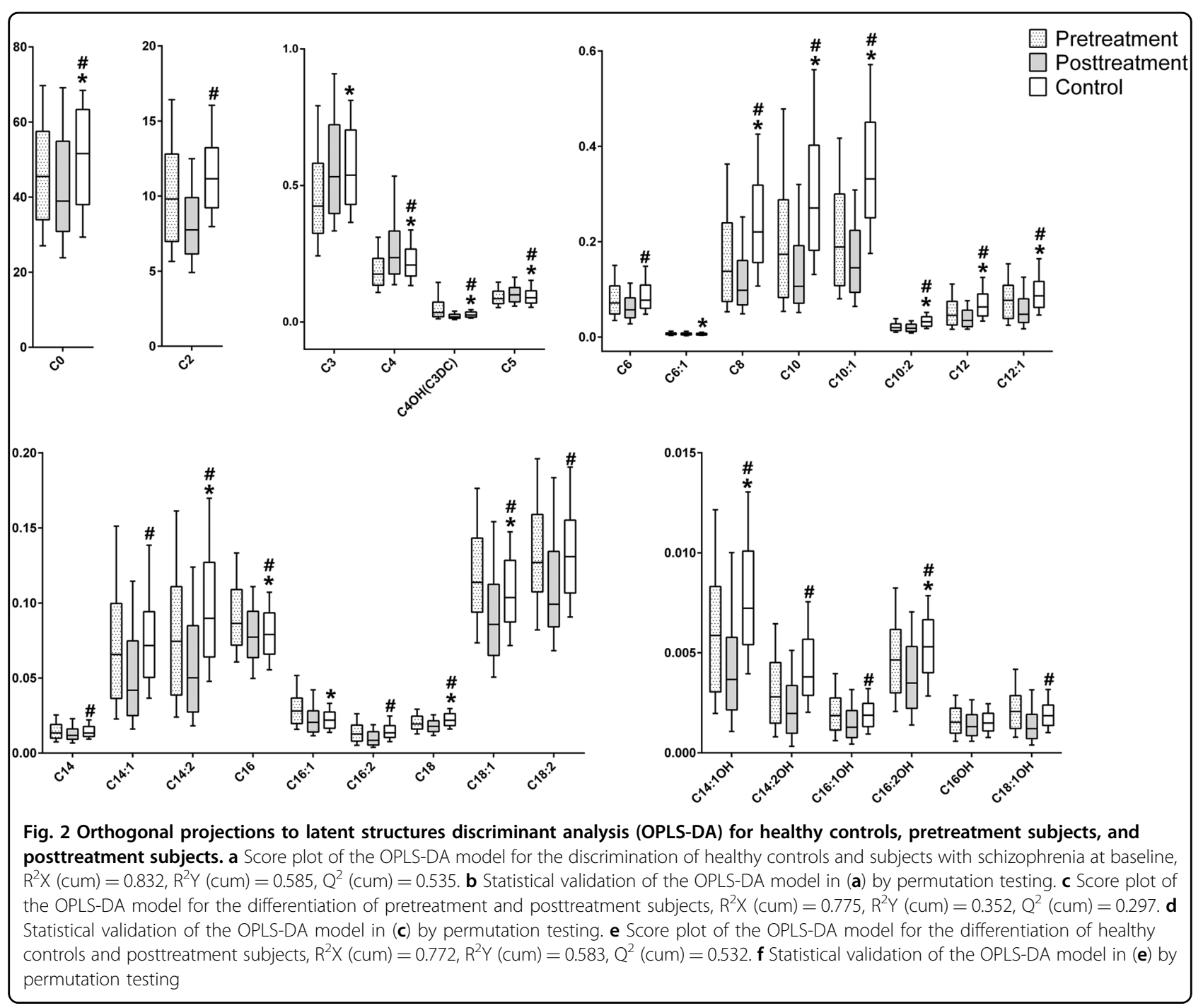

schizophrenia within a Chinese population. Our results provide a basis for future studies to determine the role of these mediators in underlying molecular processes and possibly to inform the diagnosis of schizophrenia. When acyl-carnitine profiles were compared between schizophrenia subjects and healthy controls, significantly higher levels of $\mathrm{C} 4-\mathrm{OH}(\mathrm{C} 3-\mathrm{DC})$ and $\mathrm{C} 16: 1$ were found, while lower concentrations of $\mathrm{C} 3, \mathrm{C} 8, \mathrm{C} 10, \mathrm{C} 10: 1, \mathrm{C} 10: 2, \mathrm{C} 12$, $\mathrm{C} 14: 1-\mathrm{OH}, \mathrm{C} 14: 2$, and $\mathrm{C} 14: 2-\mathrm{OH}$ were presented in the subject group after controlling for age, sex, BMI, smoking, and drinking. After the 8-week clinical treatment with mixed antipsychotic medications, all detected acylcarnitines were significantly different between the two groups. After selection by OPLS-DA VI $P$ value $>1.0$, elevations in a set of short-chain acyl-carnitines (C3 and C4) were found posttreatment. Whether the observed differences are related to the pathoetiology or possible treatment effects of schizophrenia is unknown from our current study. From the observed classification performance of our ROC curve analysis, a set of acyl-carnitines demonstrated an ability to aid differentiating schizophrenia subjects at baseline and after 8-week follow-up for both pretreatment and posttreatment subjects, as well as controls, indicating that bioenergetic abnormalities exist in subjects with schizophrenia. The acyl-carnitine panel demonstrated better diagnostic performance than other diagnostic methods in psychiatry (AUC = 0.85-0.95). Functional near-infrared spectroscopy (fNIRS) is a non-invasive neuroimaging technology that maps the functions of the cerebral cortex by measuring hemodynamics and demonstrates cost-effectiveness ${ }^{34}$. For differentiating schizophrenia and bipolar disorder from major depressive disorder by fNIRS, the AUC is $0.74^{35}$. For differentiating major depressive disorder from healthy 
Table 3 Comparison of acyl-carnitines between subjects with schizophrenia at baseline vs. healthy controls, pretreatment vs. posttreatment patients, posttreatment patients vs. healthy controls

\begin{tabular}{|c|c|c|c|c|c|c|c|c|c|}
\hline \multirow[t]{3}{*}{ Category } & \multirow{2}{*}{\multicolumn{3}{|c|}{$\begin{array}{l}\text { Patients at baseline vs. } \\
\text { Healthy controls }\end{array}$}} & \multirow{2}{*}{\multicolumn{3}{|c|}{$\begin{array}{l}\text { Pretreatment vs. } \\
\text { Posttreatment patients }\end{array}$}} & \multirow{2}{*}{\multicolumn{3}{|c|}{$\begin{array}{l}\text { Posttreatment patients vs. } \\
\text { Healthy controls }\end{array}$}} \\
\hline & & & & & & & & & \\
\hline & $q^{\mathrm{a}}$ & $\mathrm{FC}^{\mathrm{b}}$ & VIP & $q^{c}$ & $\mathrm{FC}^{\mathrm{d}}$ & VIP & $q^{\mathrm{e}}$ & $\mathrm{FC}^{\mathrm{f}}$ & VIP \\
\hline $\mathrm{CO}$ & $7.87 \mathrm{E}-04$ & 0.86 & 0.96 & 0.03 & 0.84 & 0.78 & $1.22 \mathrm{E}-07$ & 0.72 & 0.93 \\
\hline C2 & 0.47 & 0.93 & 0.69 & 3.44E-08 & 0.74 & 1.18 & $6.06 \mathrm{E}-21$ & 0.68 & 1.15 \\
\hline C3 & $1.56 \mathrm{E}-10$ & 0.76 & 1.29 & 1.74E-09 & 1.31 & 1.15 & 0.92 & 1.02 & 0.67 \\
\hline C4 & $1.50 \mathrm{E}-04$ & 0.81 & 0.99 & $4.54 \mathrm{E}-11$ & 1.47 & 1.28 & $1.81 \mathrm{E}-06$ & 1.19 & 0.81 \\
\hline $\mathrm{C} 4-\mathrm{OH}(\mathrm{C} 3-\mathrm{DC})\left(10^{-2}\right)$ & 1.56E-06 & 1.39 & 1.04 & $2.53 \mathrm{E}-13$ & 0.51 & 1.27 & 1.83E-07 & 0.71 & 0.81 \\
\hline C5 $\left(10^{-2}\right)$ & 0.08 & 0.95 & 0.73 & $1.93 \mathrm{E}-03$ & 1.2 & 0.83 & 0.21 & 1.11 & 0.65 \\
\hline C6 $\left(10^{-2}\right)$ & 0.01 & 0.92 & 0.86 & $7.05 E-04$ & 0.77 & 0.97 & $5.95 \mathrm{E}-12$ & 0.71 & 0.98 \\
\hline C6:1 $\left(10^{-2}\right)$ & 0.01 & 1.11 & 0.72 & $1.85 \mathrm{E}-03$ & 0.87 & 0.72 & 0.39 & 0.94 & 0.6 \\
\hline C8 $\left(10^{-2}\right)$ & $3.39 \mathrm{E}-11$ & 0.59 & 1.34 & $3.79 E-03$ & 0.66 & 0.93 & $2.44 \mathrm{E}-24$ & 0.39 & 1.25 \\
\hline $\mathrm{C} 10\left(10^{-2}\right)$ & $3.28 \mathrm{E}-11$ & 0.62 & 1.33 & $7.05 E-04$ & 0.54 & 0.97 & $4.87 \mathrm{E}-26$ & 0.35 & 1.3 \\
\hline C10:1 $\left(10^{-2}\right)$ & $1.60 \mathrm{E}-17$ & 0.56 & 1.55 & $2.43 \mathrm{E}-04$ & 0.66 & 1.02 & $3.75 E-33$ & 0.38 & 1.37 \\
\hline C10:2 $\left(10^{-2}\right)$ & $2.78 \mathrm{E}-19$ & 0.59 & 1.57 & 0.04 & 0.87 & 0.79 & $1.86 \mathrm{E}-24$ & 0.52 & 1.22 \\
\hline C12 $\left(10^{-2}\right)$ & $3.48 \mathrm{E}-08$ & 0.69 & 1.19 & 0.01 & 0.71 & 0.9 & $2.52 E-18$ & 0.52 & 1.15 \\
\hline C12:1 $\left(10^{-2}\right)$ & $1.25 E-03$ & 0.91 & 0.96 & 1.63E-04 & 0.56 & 1.03 & $3.10 E-15$ & 0.53 & 1.12 \\
\hline C14 $\left(10^{-2}\right)$ & 0.58 & 1.01 & 0.74 & 7.84E-03 & 0.86 & 0.85 & $2.20 \mathrm{E}-04$ & 0.87 & 0.84 \\
\hline C14:1 $\left(10^{-2}\right)$ & 0.07 & 0.93 & 0.82 & $1.59 E-05$ & 0.59 & 1.09 & $4.00 E-13$ & 0.55 & 1.07 \\
\hline C14:1-OH $\left(10^{-2}\right)$ & $6.33 \mathrm{E}-07$ & 0.8 & 1.09 & 4.30E-05 & 0.61 & 1.04 & $2.75 E-20$ & 0.49 & 1.19 \\
\hline C14:2 $\left(10^{-2}\right)$ & 7.71E-05 & 0.84 & 1.02 & 1.06E-04 & 0.59 & 1.08 & $3.20 E-18$ & 0.5 & 1.16 \\
\hline $\mathrm{C} 14: 2-\mathrm{OH}\left(10^{-2}\right)$ & 3.63E-09 & 0.72 & 1.2 & $7.05 E-04$ & 0.68 & 0.96 & $1.02 E-19$ & 0.49 & 1.17 \\
\hline C16 $\left(10^{-2}\right)$ & $1.04 \mathrm{E}-03$ & 1.09 & 0.89 & $8.04 \mathrm{E}-07$ & 0.82 & 0.87 & 0.15 & 0.96 & 0.68 \\
\hline C16:1 $\left(10^{-2}\right)$ & $8.73 E-06$ & 1.28 & 1.03 & $1.14 \mathrm{E}-08$ & 0.68 & 1.1 & 0.03 & 0.91 & 0.78 \\
\hline $\mathrm{C} 16: 1-\mathrm{OH}\left(10^{-2}\right)$ & 0.97 & 0.98 & 0.71 & $9.36 \mathrm{E}-05$ & 0.68 & 1 & $1.22 \mathrm{E}-07$ & 0.68 & 0.91 \\
\hline C16:2 $\left(10^{-2}\right)$ & 0.21 & 1.04 & 0.79 & $4.34 E-07$ & 0.59 & 1.17 & $3.10 E-15$ & 0.6 & 1.11 \\
\hline $\mathrm{C} 16: 2-\mathrm{OH}\left(10^{-2}\right)$ & 0.01 & 0.89 & 0.8 & $2.20 \mathrm{E}-04$ & 0.71 & 0.97 & $2.03 E-12$ & 0.64 & 1.03 \\
\hline $\mathrm{C} 16-\mathrm{OH}\left(10^{-2}\right)$ & 0.53 & 1.00 & 0.68 & 0.02 & 0.87 & 0.78 & $7.12 \mathrm{E}-05$ & 0.87 & 0.75 \\
\hline $\mathrm{C} 18\left(10^{-2}\right)$ & $1.25 \mathrm{E}-03$ & 0.89 & 0.88 & $9.99 E-08$ & 0.86 & 0.83 & $3.10 \mathrm{E}-15$ & 0.76 & 1.08 \\
\hline C18:1 $\left(10^{-2}\right)$ & $7.87 \mathrm{E}-03$ & 1.12 & 0.86 & $2.53 \mathrm{E}-13$ & 0.67 & 1.16 & $1.22 E-07$ & 0.79 & 0.89 \\
\hline C18:1-OH $\left(10^{-2}\right)$ & 0.94 & 1.11 & 0.71 & 1.37E-06 & 0.57 & 1.11 & $5.91 \mathrm{E}-10$ & 0.63 & 0.94 \\
\hline C18:2 $\left(10^{-2}\right)$ & 0.94 & 0.98 & 0.55 & $2.19 E-07$ & 0.74 & 0.84 & $1.47 \mathrm{E}-05$ & 0.76 & 0.76 \\
\hline
\end{tabular}

${ }^{\mathrm{a}, \mathrm{e}} q$ values were FDR corrections for $p$-values which were calculated from two-tailed independent $t$-test of $\log _{10}$-transformed data

${ }^{b}$ Fold changes were calculated as the ratios of median metabolite levels (patients at baseline/healthy controls)

${ }^{c} q$ values were FDR corrections for $p$ values which were calculated from two-tailed paired $t$-test of $\log _{10}$-transformed data

${ }^{d}$ Fold changes were calculated as the ratios of median metabolite levels (posttreatment patients/pretreatment patients)

$\mathrm{f}_{\mathrm{f}}$ old changes were calculated as the ratios of median metabolite levels (posttreatment patients/healthy controls)

controls by urine monoamine panel, the AUC is $0.66^{36}$. Consequently, exploring the disrupted acyl-carnitine metabolism from our current results may play a critical role in helping us to deepen our understanding on related bioenergetic pathways of schizophrenia.

Acyl-carnitines are amphiphilic molecules and facilitate mobility throughout cells, which play an essential role in 
Table 4 GLM and ROC for comparison of selected acyl-carnitines levels between subjects with schizophrenia at baseline vs. healthy controls, pretreatment vs. posttreatment patients, posttreatment patients vs. healthy controls

\begin{tabular}{|c|c|c|c|c|c|c|c|c|c|}
\hline \multirow[t]{2}{*}{ Variables } & \multirow{2}{*}{$\begin{array}{l}\text { Acyl-carnitines } \\
(\mu \mathrm{mol} / \mathrm{L}) ;\end{array}$} & \multirow[t]{2}{*}{ median (IQR) } & \multicolumn{3}{|l|}{ GLM } & \multicolumn{4}{|l|}{ ROC } \\
\hline & & & $\beta$ & $95 \% \mathrm{Cl}$ & $Z^{*}$ & AUROC & $95 \% \mathrm{Cl}$ & Sensitivity & Specificit \\
\hline \multicolumn{10}{|c|}{ Patients at baseline $(n=225)$ vs. Healthy controls $(n=175)$} \\
\hline C3 & $0.42(0.31-0.59)$ & $0.55(0.45-0.72)$ & $-0.857^{\mathrm{a}}$ & $-1.113,-0.601$ & -6.56 & 0.692 & $0.640,0.743$ & 0.714 & 0.622 \\
\hline $\begin{array}{l}\mathrm{C} 4-\mathrm{OH}(\mathrm{C} 3-\mathrm{DC}) \\
\left(10^{-2}\right)\end{array}$ & $3.57(1.9-7.85)$ & $2.56(1.79-3.68)$ & $0.317^{\mathrm{a}}$ & $0.186,0.448$ & 4.75 & 0.624 & $0.569,0.679$ & 0.467 & 0.834 \\
\hline C8 $\left(10^{-2}\right)$ & $13.68(7.25-24.2)$ & $23.1(15.98-34.69)$ & $-0.43^{\mathrm{a}}$ & $-0.569,-0.29$ & -6.04 & 0.702 & $0.651,0.752$ & 0.789 & 0.569 \\
\hline $\mathrm{C} 10\left(10^{-2}\right)$ & $17.2(7.86-29.64)$ & $\begin{array}{l}27.84 \\
(18.44-41.46)\end{array}$ & $-0.421^{\mathrm{a}}$ & $-0.553,-0.288$ & -6.22 & 0.696 & $0.645,0.747$ & 0.829 & 0.507 \\
\hline C10:1 $\left(10^{-2}\right)$ & $18.9(10.54-30.6)$ & $\begin{array}{l}33.97 \\
(25.19-46.99)\end{array}$ & $-0.633^{\mathrm{a}}$ & $-0.785,-0.482$ & -8.21 & 0.755 & $0.708,0.801$ & 0.777 & 0.640 \\
\hline C10:2 $\left(10^{-2}\right)$ & $1.95(1.34-2.77)$ & $3.3(2.31-4.46)$ & $-0.852^{a}$ & $-1.036,-0.668$ & -9.09 & 0.763 & $0.717,0.808$ & 0.834 & 0.560 \\
\hline $\mathrm{C} 12\left(10^{-2}\right)$ & $4.49(2.42-7.55)$ & $6.48(4.57-9.3)$ & $-0.42^{\mathrm{a}}$ & $-0.573,-0.266$ & -5.35 & 0.657 & $0.605,0.71$ & 0.714 & 0.547 \\
\hline $\mathrm{C} 14: 1-\mathrm{OH}\left(10^{-2}\right)$ & $0.59(0.3-0.86)$ & $0.74(0.54-1.03)$ & $-0.451^{\mathrm{a}}$ & $-0.61,-0.292$ & -5.57 & 0.633 & $0.579,0.686$ & 0.617 & 0.564 \\
\hline C14:2 $\left(10^{-2}\right)$ & 7.58(3.75-11.53) & $8.98(6.42-12.8)$ & $-0.341^{\mathrm{a}}$ & $-0.505,-0.177$ & -4.08 & 0.607 & $0.552,0.662$ & 0.789 & 0.422 \\
\hline $\mathrm{C} 14: 2-\mathrm{OH}\left(10^{-2}\right)$ & $0.28(0.15-0.45)$ & $0.39(0.29-0.58)$ & $-0.453^{\mathrm{a}}$ & $-0.587,-0.318$ & -6.61 & 0.664 & $0.611,0.716$ & 0.743 & 0.533 \\
\hline C16:1 $\left(10^{-2}\right)$ & $2.81(1.96-3.82)$ & $2.19(1.65-2.77)$ & $0.509^{\mathrm{a}}$ & $0.277,0.741$ & 4.31 & 0.658 & $0.605,0.711$ & 0.538 & 0.743 \\
\hline \multicolumn{10}{|c|}{ Pretreatment ( $n=156)$ vs. Posttreatment patients ( $n=156)$} \\
\hline$C 2$ & $10.29(6.92-15.18)$ & $7.62(5.98-9.78)$ & $0.145^{\mathrm{b}}$ & $0.098,0.191$ & 6.13 & 0.677 & $0.618,0.736$ & 0.628 & 0.628 \\
\hline C3 & $0.42(0.31-0.58)$ & $0.55(0.40-0.77)$ & $-0.136^{b}$ & $-0.175,-0.096$ & -6.78 & 0.669 & $0.610,0.729$ & 0.654 & 0.622 \\
\hline C4 & $0.17(0.13-0.23)$ & $0.25(0.18-0.40)$ & $-0.213^{b}$ & $-0.269,-0.157$ & -7.5 & 0.708 & $0.651,0.766$ & 0.603 & 0.718 \\
\hline $\begin{array}{l}\mathrm{C} 4-\mathrm{OH}(\mathrm{C} 3-\mathrm{DC}) \\
\left(10^{-2}\right)\end{array}$ & $3.62(1.95-7.81)$ & $1.83(1.34-2.79)$ & $0.349^{b}$ & $0.268,0.429$ & 8.47 & 0.734 & $0.678,0.79$ & 0.622 & 0.769 \\
\hline C10:1 $\left(10^{-2}\right)$ & $19.78(10.51-31.22)$ & 13.07(8.35-21.66) & $0.148^{\mathrm{b}}$ & $0.073,0.223$ & 3.89 & 0.632 & $0.57,0.693$ & 0.590 & 0.603 \\
\hline C12:1 $\left(10^{-2}\right)$ & $8.38(3.97-11.83)$ & $4.67(2.75-8.13)$ & $0.162^{b}$ & $0.083,0.241$ & 4.02 & 0.64 & $0.578,0.701$ & 0.571 & 0.699 \\
\hline C14:1 $\left(10^{-2}\right)$ & $6.85(3.63-10.23)$ & $4.01(2.3-7.23)$ & $0.188^{b}$ & $0.109,0.267$ & 4.67 & 0.655 & $0.594,0.715$ & 0.609 & 0.660 \\
\hline $\mathrm{C} 14: 1-\mathrm{OH}\left(10^{-2}\right)$ & $0.59(0.28-0.83)$ & $0.36(0.2-0.57)$ & $0.191^{b}$ & $0.106,0.276$ & 4.41 & 0.646 & $0.585,0.708$ & 0.603 & 0.705 \\
\hline C14:2 $\left(10^{-2}\right)$ & $7.62(3.68-11.4)$ & $4.5(2.48-8.41)$ & $0.173^{b}$ & $0.091,0.254$ & 4.15 & 0.641 & $0.58,0.703$ & 0.609 & 0.635 \\
\hline C16:1 $\left(10^{-2}\right)$ & $2.92(1.97-3.98)$ & $1.99(1.34-2.77)$ & $0.159^{b}$ & $0.11,0.208$ & 6.38 & 0.696 & $0.637,0.754$ & 0.705 & 0.603 \\
\hline $\mathrm{C} 16: 1-\mathrm{OH}\left(10^{-2}\right)$ & $0.19(0.11-0.3)$ & $0.13(0.07-0.21)$ & $0.192^{b}$ & $0.102,0.281$ & 4.2 & 0.64 & $0.579,0.701$ & 0.705 & 0.526 \\
\hline C16:2 $\left(10^{-2}\right)$ & 1.37(0.83-1.9) & $0.81(0.48-1.4)$ & $0.202^{b}$ & $0.13,0.274$ & 5.53 & 0.677 & $0.618,0.736$ & 0.609 & 0.654 \\
\hline C18:1 $\left(10^{-2}\right)$ & $12.23(9.84-15.07)$ & $8.2(5.62-11.2)$ & $0.159^{b}$ & $0.123,0.196$ & 8.58 & 0.734 & $0.678,0.79$ & 0.788 & 0.615 \\
\hline C18:1-OH $\left(10^{-2}\right)$ & $0.21(0.12-0.3)$ & $0.12(0.06-0.19)$ & $0.249^{b}$ & $0.156,0.342$ & 5.24 & 0.688 & $0.629,0.746$ & 0.712 & 0.577 \\
\hline \multicolumn{10}{|c|}{ Posttreatment patients ( $n=156)$ vs. Healthy controls $(n=175)$} \\
\hline C2 & $7.62(5.98-9.78)$ & $11.16(9.26-13.24)$ & $-1.404^{c}$ & $-1.696,-1.112$ & -9.44 & 0.788 & $0.739,0.838$ & 0.857 & 0.628 \\
\hline C8 $\left(10^{-2}\right)$ & $9.11(6.19-16.04)$ & $23.1(15.98-34.69)$ & $-0.731^{c}$ & $-0.864,-0.598$ & -10.76 & 0.822 & $0.776,0.868$ & 0.777 & 0.737 \\
\hline $\mathrm{C} 10\left(10^{-2}\right)$ & $9.61(6.58-18.74)$ & $\begin{array}{l}27.84 \\
(18.44-41.46)\end{array}$ & $-0.702^{c}$ & $-0.823,-0.582$ & -11.44 & 0.827 & $0.781,0.872$ & 0.834 & 0.705 \\
\hline C10:1 $\left(10^{-2}\right)$ & $13.07(8.35-21.66)$ & $\begin{array}{l}33.97 \\
(25.19-46.99)\end{array}$ & $-0.866^{c}$ & $-0.993,-0.74$ & -13.4 & 0.867 & $0.828,0.906$ & 0.806 & 0.827 \\
\hline
\end{tabular}


Table 4 continued

\begin{tabular}{|c|c|c|c|c|c|c|c|c|c|}
\hline \multirow[t]{2}{*}{ Variables } & \multirow{2}{*}{$\begin{array}{l}\text { Acyl-carnitines } \\
(\mu \mathrm{mol} / \mathrm{L}) ;\end{array}$} & \multirow[t]{2}{*}{ median (IQR) } & \multicolumn{3}{|l|}{ GLM } & \multicolumn{4}{|l|}{ ROC } \\
\hline & & & $\beta$ & $95 \% \mathrm{Cl}$ & $Z^{*}$ & AUROC & $95 \% \mathrm{Cl}$ & Sensitivity & Specificity \\
\hline C10:2 $\left(10^{-2}\right)$ & $1.71(1.06-2.66)$ & $3.3(2.31-4.46)$ & $-0.949^{c}$ & $-1.114,-0.783$ & -11.22 & 0.808 & $0.762,0.854$ & 0.789 & 0.699 \\
\hline C12 $\left(10^{-2}\right)$ & $3.35(2.05-5.62)$ & $6.48(4.57-9.3)$ & $-0.718^{c}$ & $-0.871,-0.565$ & -9.2 & 0.776 & $0.725,0.826$ & 0.720 & 0.718 \\
\hline C12:1 $\left(10^{-2}\right)$ & $4.67(2.75-8.13)$ & $8.74(6.23-12)$ & $-0.634^{c}$ & $-0.792,-0.476$ & -7.88 & 0.74 & $0.685,0.795$ & 0.817 & 0.596 \\
\hline C14:1 $\left(10^{-2}\right)$ & $4.01(2.3-7.23)$ & $7.23(5.04-9.51)$ & $-0.578^{c}$ & $-0.734,-0.423$ & -7.29 & 0.718 & $0.662,0.775$ & 0.783 & 0.590 \\
\hline $\mathrm{C} 14: 1-\mathrm{OH}\left(10^{-2}\right)$ & $0.36(0.2-0.57)$ & $0.74(0.54-1.03)$ & $-0.659^{c}$ & $-0.789,-0.528$ & -9.88 & 0.787 & $0.737,0.837$ & 0.766 & 0.737 \\
\hline C14:2 $\left(10^{-2}\right)$ & $4.5(2.48-8.41)$ & $8.98(6.42-12.8)$ & $-0.659^{c}$ & $-0.804,-0.514$ & -8.93 & 0.761 & $0.708,0.814$ & 0.789 & 0.654 \\
\hline $\mathrm{C} 14: 2-\mathrm{OH}\left(10^{-2}\right)$ & $0.19(0.09-0.34)$ & $0.39(0.29-0.58)$ & $-0.547^{c}$ & $-0.658,-0.437$ & -9.71 & 0.779 & $0.728,0.831$ & 0.760 & 0.699 \\
\hline C16:2 $\left(10^{-2}\right)$ & $0.81(0.48-1.4)$ & $1.36(1.04-1.84)$ & $-0.668^{c}$ & $-0.835,-0.5$ & -7.81 & 0.739 & $0.684,0.794$ & 0.714 & 0.686 \\
\hline $\mathrm{C} 16: 2-\mathrm{OH}\left(10^{-2}\right)$ & $0.34(0.22-0.53)$ & $0.53(0.4-0.68)$ & $-0.683^{c}$ & $-0.861,-0.505$ & -7.52 & 0.718 & $0.662,0.775$ & 0.720 & 0.660 \\
\hline $\mathrm{C} 18\left(10^{-2}\right)$ & $1.69(1.28-2.11)$ & $2.22(1.84-2.66)$ & $-1.458^{c}$ & $-1.785,-1.132$ & -8.76 & 0.738 & $0.685,0.792$ & 0.686 & 0.641 \\
\hline
\end{tabular}

$\beta$ regression coefficient, $\mathrm{Cl}$ confidence interval

*All $P$ values have FDR-corrected statistical significance

${ }_{a}, c \beta$ was calculated from GLM with adjustment for age, gender, BMI, current smoking and current drinking.

${ }^{\mathrm{b}} \beta$ was calculated from GLM without adjustment

the transport of activated long-chain fatty acids from the cytosol across the mitochondrial membrane toward the mitochondrial matrix, where $\beta$-oxidation takes place ${ }^{37,38}$. Similar to small and water-soluble molecules, free carnitines and short-chain acyl-carnitines are easily transported across the plasma membrane and can be used to deliver acyl-groups to various locations with wide ranging functions ${ }^{11,39}$. However, medium-chain and long-chain acyl-carnitines require transporters to cross the plasma membrane, which may result in regulated biological functions ${ }^{11,40}$. In our current study, strong positive correlations among most medium-chain and long-chain acylcarnitines were found with each other (from C6 to C18, Fig. S3). Our findings suggest that changes in individual acyl-carnitines possibly correlate with each other, especially medium-chain and long-chain acyl-carnitines. Furthermore, acyl-carnitines have important roles in the regulation of cellular energetics and mitochondrial functioning, including roles in the central nervous system $(\mathrm{CNS})^{41,42}$. Previous systematic reviews reported that neuropsychiatric disorders, such as Alzheimer's disease and autism, often share similar pathologies in mitochondrial dysfunction ${ }^{43}$ regarding energetic metabolic abnormalities.

The comparison between individuals with schizophrenia and healthy controls indicates that nearly all the medium-chain and long-chain acyl-carnitines were lower in the subject group, which may be caused by the activation or inhibition of different transporters in the plasma membrane. Historically, the function of carnitines and their role in the pathophysiology of schizophrenia are not completely clarified. Certain studies have pointed out that acyl-carnitines which have high rates of $\beta$-oxidation are found to increase antioxidant activity, regulate immune functions ${ }^{44}$, stimulate the activity of certain proteins and enzymes, and enhance cholinergic neurotransmission ${ }^{40}$. On the other hand, other studies suggest that acylcarnitines are harmful fatty acid intermediates, which have a role in inducing inhibition of oxidative phosphorylation and increasing apoptosis ${ }^{45}$. Past research also reports that carnitines can be altered under different metabolic conditions ${ }^{26}$; this was illustrated in a population-based study with diabetes that demonstrated carnitines potentially are associated with the pathogenesis of insulin resistance caused by mitochondrial stress, including incomplete fatty acid oxidation and mitochondrial lipid overload ${ }^{18}$. Furthermore, previous studies indicate that treatment with long-chain acyl-carnitines induced insulin resistance and oxidative stress in human skeletal muscles ${ }^{46}$. The conflicting evidence pertaining to acyl-carnitines further sheds light on the fact that more research is needed on this important topic.

After the 8-week follow-up point, a panel of mediumchain and long-chain acyl-carnitines were lower in posttreatment subjects than they were at baseline. Perhaps the observed downregulation of these carnitines after treatment administration is indicative of altered metabolic pathways in energy metabolism, which has been observed in conditions with increased catabolism (e.g., senile dementia, metabolic nerve diseases, tuberculosis, myopathies and cardiomyopathies ${ }^{47}$. This decrease is also potentially a result of several antipsychotic drugs or mood 


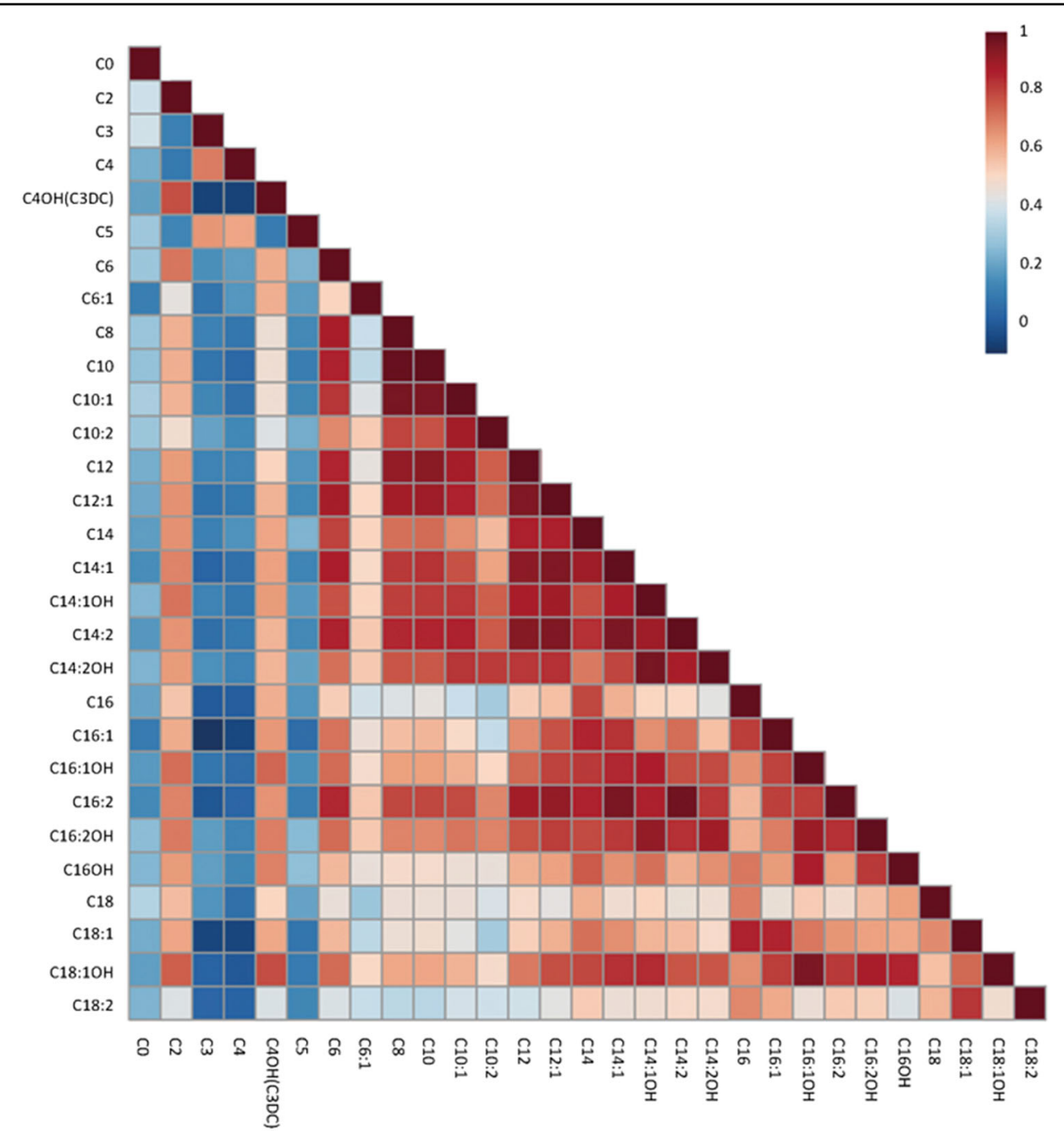

Fig. 3 Correlations between the acyl-carnitine levels. Red and blue represent positive and negative correlations, respectively

stabilizers (e.g., valproic acid) that are involved in secondary carnitine deficiency which have been found to reduce levels of acyl-carnitines ${ }^{48,49}$.

Attention needs to be drawn to the increase of $\mathrm{C} 3$ and C4 after 8-week treatment (two short-chain acyl-carnitines) due to the possibility of their involvement in different metabolic pathways compared to the other carnitines. Fatty acids can across the plasma membrane with the fatty acid transport proteins. After transportation into the cell by fatty acid transporters, fatty acids are activated by esterification to Acyl-Coenzyme A (AcylCoA). Subsequently, Carnitine palmitoyltransferase 1 (CPT1) catalyzes transfer acyl groups from Acyl-CoA to carnitine to produce medium-chain and long-chain acylcarnitines and free coenzyme A (CoA) under normal conditions $^{50}$. The resulting acyl-carnitines are transported across the inner mitochondrial membrane into the mitochondrion matrix ${ }^{51,52}$. Furthermore, carnitine acetyltransferase (CAT) catalyzes the synthesis of short-chain acyl-carnitines from Acyl-CoA, which is located on the inner mitochondrial membrane, microsomes, and peroxisomes ${ }^{53,54}$. Elevations in a set of short-chain acyl-carnitines ( $\mathrm{C} 3$ and $\mathrm{C} 4)$ in posttreatment subjects speculates the dysfunction of CPT1 and CAT; or the deficiency of related acyl-CoA (such as propionyl-CoA carboxylase, isobutyryl-CoA dehydrogenase, isovaleryl-CoA dehydrogenase, and succinyl-CoA synthetase) which also have proven to cause the downregulation of free carnitines ${ }^{11}$.

Acyl-carnitines have previously shown to be reliable biomarkers in the diagnosis or prediction of disease ${ }^{18,55}$ such as type 2 diabetes and atypical myopathy. Moreover, other lines of research indicate that acyl-carnitine supplementation can be considered as a potential therapeutic strategy across several neuropsychiatric disorders (i.e., 
schizophrenia, autism, depression, etc.) with unique neuroprotective effects including anti-inflammatory and antioxidant benefits ${ }^{27,43}$. Our research results and past published evidence uniformly indicate that acyl-carnitine profiles may be a useful indicator of metabolic changes in schizophrenia, additionally acyl-carnitine supplementation therapies may have accompanying positive potential in the treatment of schizophrenia.

Strengths of our study include the incorporation of high-dimensional datasets with validated profiling tests in a schizophrenia population such that acyl-carnitines with abnormalities in cellular bioenergetics are characterized in schizophrenia. However, the study has several limitations. First, only $69.3 \%$ of subjects with schizophrenia were successfully followed. The clinical characteristics and acetyl-carnitines of the followed subjects may differ with those lost to follow-up. Secondly, an 8-week followup duration may not be sufficient to observe long-term metabolic and clinical changes (e.g., the development of other medical co-morbidities). Furthermore, given the limited sample size and the heterogeneity of the antipsychotic drugs administered, results should be interpreted with caution. There is also the possibility that changes in plasma acyl-carnitine levels can result from pharmacological intervention. In such cases, the role of plasma acyl-carnitine levels in the etiology of schizophrenia pathogenesis may be limited. Further research on specific antipsychotics are needed to explore pharmacological effects on acyl-carnitines in the future. Finally, the presentation of schizophrenia can be heterogeneous, consequently, our results may not fully capture metabolomic profiles of all presentations of schizophrenia. The reported results in our study provide rationale for the exploration of acyl-carnitines in schizophrenia beyond the Chinese population, as well as larger sample cohorts with longer longitudinal durations.

\section{Conclusion}

In conclusion, our study suggests that acyl-carnitines provide a potential pathway in the pathoetiology of schizophrenia. Acyl-carnitines present novel treatment and screening opportunities for patients with schizophrenia. The current results indicating that bioenergetic abnormalities exist in subjects with schizophrenia. The reported results contained herein warrant further exploration of acyl-carnitine profiles in different populations and large sample cohorts with longer longitudinal durations.

\section{Acknowledgements}

The authors alone are responsible for the content and writing of the paper. We thank team members for their support and contributions to this study. We thank Dr. Chuanbo Zhang and other medical staff from Weifang Mental Health Center for their assistance with subject recruitment, follow-up and blood sample collection. We also thank Beijing Omics Bio-tech Co., Ltd for their kind help in data collection for this study. This work was supported by the Medicine Interdisciplinary Seed Fund (BMU20140435) by Health Science Center, Peking
University. The supporting foundation was not involved in study design, the collection, analysis, or interpretation of data, writing of the report; or in the decision to submit the article for publication.

\section{Author details}

'Department of Laboratorial Science and Technology, School of Public Health, Peking University, Beijing 100191, P. R. China. ${ }^{2}$ Mood Disorders Psychopharmacology Unit, Toronto Western Hospital, University Health Network, Toronto, ON, Canada. ${ }^{3}$ Department of Psychiatry, Federal University of São Paulo, São Paulo, SP, Brazil. "Brain and Cognition Discovery Foundation, Toronto, ON, Canada. ${ }^{5}$ Beijing Key Laboratory of Toxicological Research and Risk Assessment for Food Safety, Beijing 100191, P.R. China. ${ }^{6}$ Peking University Medical and Health Analysis Center, Peking University, Beijing 100191, P.R. China

\section{Author contributions}

J.Y.W., B.C., and D.F.W. conceived and designed the study; B.C., D.F.W., J.Z., and N.H.H. collected the data and performed the statistical analysis; B.C., Z.P., E.B., R. S.M., J.Y.W., and D.F.W. contributed to the discussion; J.Y.W., Z.P., R.S.M., E.B., M. S., R.B.M., and N.M. revised the paper. All authors have read and approved the final version of this article.

\section{Conflict of interest}

The authors declare that they have no conflict of interest.

\section{Ethical approval}

This study was reviewed and approved by the Ethics Review Committee of Public Health at Peking University Health Science Center (IRB00001052-14071).

\section{Publisher's note}

Springer Nature remains neutral with regard to jurisdictional claims in published maps and institutional affiliations.

Supplementary Information accompanies this paper at (https://doi.org/ 10.1038/s41398-018-0353-x).

Received: 14 May 2018 Revised: 18 August 2018 Accepted: 8 November 2018

Published online: 17 January 2019

\section{References}

1. Saha, S., Chant, D., Welham, J. \& McGrath, J. A systematic review of the prevalence of schizophrenia. PLoS. Med. 2, e141 (2005).

2. Shi, J. et al. Common variants on chromosome 6p22.1 are associated with schizophrenia. Nature 460, 753-757 (2009).

3. Addington, J. \& Addington, D. Neurocognitive and social functioning in schizophrenia. Schizophr. Bull. 25, 173-182 (1999).

4. Grimm, O., Kaiser, S., Plichta, M. M. \& Tobler, P. N. Altered reward anticipation: potential explanation for weight gain in schizophrenia? Neurosci. Biobehav. Rev. 75, 91-103 (2017).

5. Annamalai, A., Kosir, U. \& Tek, C. Prevalence of obesity and diabetes in patients with schizophrenia. World J. Diabetes 8, 390-396 (2017).

6. Suvitaival, T. et al. Serum metabolite profile associates with the development of metabolic co-morbidities in first-episode psychosis. Transl. Psychiatry 6, e951 (2016).

7. Greenhalgh, A. M. et al. Meta-analysis of glucose tolerance, insulin, and insulin resistance in antipsychotic-naive patients with nonaffective psychosis. Schizophr. Res. 179, 57-63 (2017).

8. Kirkpatrick, B., Miller, B. J., Garcia-Rizo, C., Fernandez-Egea, E. \& Bernardo, M. Is abnormal glucose tolerance in antipsychotic-naive patients with nonaffective psychosis confounded by poor health habits? Schizophr. Bull. 38, 280-284 (2012).

9. Vaz, F. M. \& Wanders, R. J. Carnitine biosynthesis in mammals. Biochem. J. 361, 417-429 (2002). (Pt 3).

10. Coskun, A. \& Dogan, M. Liver L-carnitine biosynthesis in non-pregnant, pregnant and lactating rabbits. Res. Commun. Mol. Pathol. Pharmacol. 111, 211-223 (2002). 
11. Jones, L. L., McDonald, D. A. \& Borum, P. R. Acylcarnitines: role in brain. Prog. Lipid Res. 49, 61-75 (2010).

12. Costa, C. G. et al. Quantitative analysis of plasma acylcarnitines using gas chromatography chemical ionization mass fragmentography. J. Lipid Res. 38, 173-182 (1997).

13. Schonfeld, P. \& Wojtczak, L. Short- and medium-chain fatty acids in energy metabolism: the cellular perspective. J. Lipid Res. 57, 943-954 (2016).

14. Noland, R. C. et al. Carnitine insufficiency caused by aging and overnutrition compromises mitochondrial performance and metabolic control. J. Biol. Chem. 284, 22840-22852 (2009).

15. Smeland, O. B., Meisingset, T. W., Borges, K. \& Sonnewald, U. Chronic acetyl-Lcarnitine alters brain energy metabolism and increases noradrenaline and serotonin content in healthy mice. Neurochem. Int. 61, 100-107 (2012).

16. Cavedon, C. T. et al. Age-related variations in acylcarnitine and free carnitine concentrations measured by tandem mass spectrometry. Clin. Chem. 51, 745-752 (2005).

17. Wong, S. et al. Acylcarnitine profile in thyroid disease. Clin. Biochem. 46, 180-183 (2013).

18. Sun, L. et al. Early prediction of developing type 2 diabetes by plasma acylcarnitines: a population-based study. Diabetes Care 39, 1563-1570 (2016).

19. Signorelli, S. S. et al. A randomised, controlled clinical trial evaluating changes in therapeutic efficacy and oxidative parameters after treatment with propionyl L-carnitine in patients with peripheral arterial disease requiring haemodialysis. Drugs Aging 23, 263-270 (2006).

20. Ciacci, C. et al. L-Carnitine in the treatment of fatigue in adult celiac disease patients: a pilot study. Dig. Liver. Dis. 39, 922-928 (2007).

21. Wang, S. M. et al. A review of current evidence for acetyl-I-carnitine in the treatment of depression. J. Psychiatr. Res. 53, 30-37 (2014).

22. Frye, R. E., Melnyk, S. \& Macfabe, D. F. Unique acyl-carnitine profiles are potential biomarkers for acquired mitochondrial disease in autism spectrum disorder. Transl. Psychiatry 3, e220 (2013).

23. Kriisa, K. et al. Profiling of acylcarnitines in first episode psychosis before and after antipsychotic treatment. J. Proteome Res. 16, 3558-3566 (2017).

24. Cuturic, M., Abramson, R. K., Breen, R. J., Edwards, A. C. \& Levy, E. E. Comparison of serum carnitine levels and clinical correlates between outpatients and acutely hospitalised individuals with bipolar disorder and schizophrenia: A cross-sectional study. World J. Biol. Psychiatry 17, 475-479 (2016).

25. Jamilian, $\mathrm{H}$. et al. Oral carnitine supplementation influences mental health parameters and biomarkers of oxidative stress in women with polycystic ovary syndrome: a randomized, double-blind, placebo-controlled trial. Gynecol. Endocrinol. 33, 442-447 (2017).

26. Cuturic, M., Abramson, R. K. Moran, R. R. \& Hardin, J. W. Clinical outcomes and low-dose levocarnitine supplementation in psychiatric inpatients with documented hypocarnitinemia: a retrospective chart review. J. Psychiatr. Pract. 16, 5-14 (2010).

27. Bruno, A. et al. Acetyl-L-carnitine augmentation of clozapine in partialresponder schizophrenia: a 12-week, open-label uncontrolled preliminary study. Clin. Neuropharmacol. 39, 277-280 (2016).

28. Muoio, D. M. \& Neufer, P. D. Lipid-induced mitochondrial stress and insulin action in muscle. Cell. Metab. 15, 595-605 (2012).

29. Bene, J. et al. Plasma carnitine ester profiles in Crohn's disease patients characterized for SLC22A4 C1672T and SLC22A5 G-207C genotypes. Br. J. Nutr. 98, 345-350 (2007).

30. Ensenauer, R. et al. In situ assay of fatty acid beta-oxidation by metabolite profiling following permeabilization of cell membranes. J. Lipid Res. 53, 1012-1020 (2012).
31. $\mathrm{Xu}, \mathrm{T}$. et al. Effects of smoking and smoking cessation on human serum metabolite profile: results from the KORA cohort study. BMC Med. 11, 60 (2013).

32. Lacruz, M. E. et al. Cardiovascular risk factors associated with blood metabolite concentrations and their alterations during a 4-year period in a populationbased cohort. Circ. Cardiovasc Genet. 9, 487-494 (2016).

33. Zheng, P. et al. Identification and validation of urinary metabolite biomarkers for major depressive disorder. Mol. \& Cell. Proteom.: MCP 12, 207-214 (2013).

34. Lai, C. Y., Ho, C. S., Lim, C. R. \& Ho, R. C. Functional near-infrared spectroscopy in psychiatry. BJPsych Adv. 23, 324-330 (2017).

35. Takizawa, R. et al. Neuroimaging-aided differential diagnosis of the depressive state. Neuroimage $\mathbf{8 5}, 498-507$ (2014).

36. Wijaya, C. S. et al. Differentiating medicated patients suffering from major depressive disorder from healthy controls by spot urine measurement of monoamines and steroid hormones. Int. J. Environ. Res. Public. Health 15, 865 (2018).

37. Kerner, J. \& Hoppel, C. Fatty acid import into mitochondria. Mol. Cell Biol. Lipids 1486, 1-17 (2000)

38. Ho, J. K., Duclos, R. I. Jr \& Hamilton, J. A. Interactions of acyl carnitines with model membranes: a (13)C-NMR study. J. Lipid Res 43, 1429-1439 (2002).

39. Beckmann, M. et al. Changes in the human plasma and urinary metabolome associated with acute dietary exposure to sucrose and the identification of potential biomarkers of sucrose intake. Mol. Nutr. Food Res. 60, 444-457 (2016).

40. Mutomba, M. C. et al. Regulation of the activity of caspases by L-carnitine and palmitoylcarnitine. FEBS Lett. 478, 19-25 (2000).

41. Virmani, A. \& Binienda, Z. Role of carnitine esters in brain neuropathology. Mol. Asp. Med. 25, 533-549 (2004).

42. Pande, S. V. A mitochondrial carnitine acylcarnitine translocase system. Proc. Natl Acad. Sci. USA 72, 883-887 (1975).

43. Moos, W. H. et al. Epigenetic treatment of neuropsychiatric disorders: autism and schizophrenia. Drug. Dev. Res. 77, 53-72 (2016).

44. Waagsbo, B. et al. Low levels of short- and medium-chain acylcarnitines in HIVinfected patients. Eur. J. Clin. Invest. 46, 408-417 (2016).

45. Liepinsh, E. et al. Long-chain acylcarnitines determine ischaemia/reperfusioninduced damage in heart mitochondria. Biochem. J. 473, 1191-1202 (2016).

46. Aguer, C. et al. Acylcarnitines: potential implications for skeletal muscle insulin resistance. Faseb. J. 29, 336-345 (2015).

47. Evangeliou, A. \& Vlassopoulos, D. Carnitine metabolism and deficit--when supplementation is necessary? Curr. Pharm. Biotechnol. 4, 211-219 (2003).

48. Lheureux, P. E. \& Hantson, P. Carnitine in the treatment of valproic acidinduced toxicity. Clin. Toxicol. (Phila.). 47, 101-111 (2009).

49. Lheureux, P. E., Penaloza, A., Zahir, S. \& Gris, M. Science review: carnitine in the treatment of valproic acid-induced toxicity - what is the evidence? Crit. Care. $\mathbf{9}$, 431-440 (2005)

50. Koves, T. R. et al. Mitochondrial overload and incomplete fatty acid oxidation contribute to skeletal muscle insulin resistance. Cell. Metab. 7, 45-56 (2008).

51. Schooneman, M. G., Vaz, F. M., Houten, S. M. \& Soeters, M. R. Acylcarnitines: reflecting or inflicting insulin resistance? Diabetes 62, 1-8 (2013).

52. Longo, N., Frigeni, M. \& Pasquali, M. Carnitine transport and fatty acid oxidation. Biochim. Biophys. Acta 1863, 2422-2435 (2016).

53. Steiber, A., Kerner, J. \& Hoppel, C. L. Carnitine: a nutritional, biosynthetic, and functional perspective. Mol. Asp. Med. 25, 455-473 (2004).

54. Chace, D. H., Kalas, T. A. \& Naylor, E. W. Use of tandem mass spectrometry for multianalyte screening of dried blood specimens from newborns. Clin. Chem. 49, 1797-1817 (2003).

55. Boemer, F. et al. Acylcarnitines profile best predicts survival in horses with atypical myopathy. PLOS. ONE. 12, e0182761 (2017). 